\title{
HIPERTENSÃO ARTERIAL E DOENÇA ARTERIAL PERIFÉRICA
}

\author{
HYPERTENSION AND PERIPHERAL ARTERIAL DISEASE
}

Maria Teresa Nogueira Bombigị, Fernando Focaccia Póvoa', Rui Póvoa

\section{RESUMO}

A hipertensão arterial é o fator mais potente no desenvolvimento da doença vascular aterosclerótica e um fator de risco importante para a doença arterial periférica (DAP) e suas complicações. A DAP é resultante do bloqueio das artérias que fornecem sangue aos membros inferiores, em geral secundário à aterosclerose. Destaca-se o aumento de casos de pacientes com DAP, juntamente com o crescimento da expectativa de vida, variando quantitativamente conforme o sexo e a comorbidade já em curso, tais como: hipertensão, diabetes, tabagismo; dislipidemia, idade, dentre outros. A maioria dos pacientes com DAP são assintomáticos ou não apresenta o sintoma mais presente: claudicação intermitente, mas quando sintomáticos estes podem referir dor, desconforto, queimação ou câimbra em membros inferiores. Para diagnóstico da DAP encontra-se como mais referenciado o índice tornozelo-braquial (ITB) com Doppler, mas também formas de se identificar a doença, por meio da angiotomografia, angioressonância magnética, arteriografia por punção direta, além do exame físico minuncioso, este sim imprescindível. A redução da pressão arterial sistólica (PAS) reduz eventos cardiovasculares e a meta atual para tratamento na DAP é de PA $\leq 140 \times 90 \mathrm{mmHg}$ (em diabéticos, considerar PA diastólica $\leq 85 \mathrm{mmHg}$ ). A terapia anti-hipertensiva deve ser administrada a pacientes com hipertensão e DAP para reduzir o risco de IM, AVC, insuficiência cardíaca e morte cardiovascular. O uso de inibidores da enzima conversora da angiotensina (IECA) ou bloqueadores dos receptores da angiotensina (BRA) são eficazes neste propósito e são drogas de escolha na hipertensão. Não há contra indicação aos betabloqueadores nestes pacientes. A cessação do tabagismo, exercícios, terapia com estatinas, terapia antiplaquetária com aspirina ou clopidogrel e, possivelmente, cilostazol em pacientes sem história de insuficiência cardíaca controlam parte dos fatores de risco, auxiliando assim a continuidade da assistência ao paciente com DAP quando da necessidade de angioplastia com stent, uso de balão arterial, cirurgia de by-pass, além da revascularização endovascular.

Descritores: Hipertensão, Doença Arterial Periférica, Fatores de Risco.

\section{ABSTRACT}

Arterial hypertension is the most potent factor in the development of atherosclerotic vascular disease and an important risk factor for peripheral arterial disease (PAD) and its complications. PAD results from the blockage of the arteries that supply blood to the lower limbs, usually secondary to atherosclerosis. The increase in cases of patients with PAD stands out, together with the increase in life expectancy, varying quantitatively according to sex and comorbidity already underway, such as: hypertension, diabetes, smoking; dyslipidemia, age, among others. Most patients with PAD are asymptomatic or do not have the most common symptom: intermittent claudication, but when symptomatic they may report pain, discomfort, burning or cramps in the lower limbs. For the diagnosis of PAD, the ankle-brachial index (ABI) with Doppler is the most referenced, but also ways to identify the disease, through angiotomography, magnetic resonance angiography, arteriography by direct puncture, in addition to detailed physical examination. The reduction in systolic blood pressure (SBP) reduces cardiovascular events and the current target for treatment in PAD is $B P \leq 140 \times 90 \mathrm{mmHg}$ (in diabetics, consider diastolic BP $\leq 85 \mathrm{mmHg}$ ). Antihypertensive therapy should be administered to patients with hypertension and PAD to reduce the risk of MI, stroke, heart failure and cardiovascular death. The use of angiotensin-converting enzyme (ACEI) inhibitors or angiotensin receptor blockers (BRA) are effective in this regard and are drugs of choice in hypertension. There is no contraindication to beta-blockers in these patients. Smoking cessation, exercise, statin therapy, antiplatelet therapy with aspirin or clopidogrel and, possibly, cilostazol in patients without a history of heart failure control part of the risk factors, thus helping the continuity of assistance to patients with PAD when the need for stent angioplasty, use of an arterial balloon, bypass surgery, in addition to endovascular revascularization.

Keywords: Hypertension, Peripheral Arterial Disease, Risk Factors.

1. Setor de Cardiopatia Hipertensiva da Disciplina de Cardiologiada Universidade Federal de São Paulo (UNIFESP) 


\section{INTRODUÇÃO}

A hipertensão arterial (HA) foi identificada como o principal fator de risco para a mortalidade e anos de vida perdidos ajustados por incapacidade. ${ }^{1}$ É o fator mais potente no desenvolvimento da doença vascular aterosclerótica e um fator de risco importante para a doença arterial periférica do membro inferior (DAP) e suas complicações.

Aproximadamente $50 \%$ dos pacientes que apresentam DAP são relatados como hipertensos. Estima-se que mais de 200 milhões de pessoas tenham DAP no mundo inteiro; houve um aumento de 25\% desde 2000 e é a terceira causa principal de morbidade cardiovascular aterosclerótica, após doença arterial coronariana (DAC) e acidente vascular cerebral (AVC). ${ }^{2}$ É, portanto, uma causa significativa de morbidade e mortalidade reconhecida como uma pandemia global. ${ }^{3}$

A DAP é uma doença aterosclerótica sistêmica que resulta do estreitamento e oclusão das artérias que fornecem sangue às pernas, geralmente devido à aterosclerose e trombose associada. ${ }^{4}$

A DAP é uma das principais causas de morbidade devido ao declínio funcional associado e perda de membros. Tanto a DAP assintomática quanto a sintomática são preditoras significativas de eventos de doença cardiovascular (DCV) e mortalidade. As evidências atuais sugerem que a DAP representa um risco de DCV equivalente ou pior do que a DAC e por isso requer um tratamento médico agressivo. ${ }^{5}$

\section{QUADRO CLÍNICO}

Os sintomas da DAP variam de nenhum para grave; as suas principais apresentações clínicas são a claudicação intermitente $(\mathrm{Cl})$ e a isquemia crítica do membro (ICM). A $\mathrm{Cl}$ é o sintoma de apresentação mais reconhecida embora se apresenta em apenas 10\% dos pacientes. Seu início é geralmente gradual e frequentemente não é percebido por muitos adultos idosos que podem atribuir seus sintomas à artrite ou à idade. Ocorre em um grupo muscular distal à oclusão arterial durante o exercício, causando dor, desconforto, queimação ou câimbra em panturrilha, coxa ou região glútea, que ocorre durante a caminhada e que é rapidamente aliviada pelo repouso. Está associada à capacidade funcional reduzida e à redução da qualidade de vida, além de altas taxas de eventos cardiovasculares, como infarto do miocárdio (IM) e AVC. ${ }^{2}$ A ICM é uma manifestação mais grave da DAP, que apresenta dor em repouso, ulceração isquêmica ou gangrena do pé. Pacientes com ICM apresentam alto risco de perda de membros e eventos vasculares fatais ou não fatais, tais como IM e AVC. ${ }^{5} \mathrm{~A}$ isquemia aguda do membro ocorre quando há uma interrupção súbita do fluxo sanguíneo para um membro, geralmente devida a uma embolia ou trombose. ${ }^{6}$ Em contraste com a ICM, que normalmente se desenvolve ao longo de um período prolongado, muitas vezes precedido por $\mathrm{Cl}$, os pacientes com isquemia aguda do membro podem não ter sintomas anteriores. A isquemia aguda do membro geralmente ameaça a viabilidade do mesmo com mais urgência do que a ICM, possivelmente devido à falta de suprimento colateral de sangue já estabelecido para este membro. ${ }^{6}$

\section{FATORES DE RISCO}

Os fatores de risco (FR) associados à DAP são semelhantes aos descritos para a DAC, incluindo aqueles marcadores de risco mais recentes, como variáveis da hemostasia ou inflamação. Entretanto, ao contrário da DAC, os acidentes nas placas ateroscleróticas, levando à isquemia aguda e crítica são muito mais raros. ${ }^{7}$ Os principais FR associados a esta condição são:

- Colesterol elevado

- Diabetes

- Doença arterial coronária

- Hipertensão arterial

- Doença renal dialítica

- Tabagismo

- Doença cerebrovascular

- Histórico familiar

- Sedentarismo

- Obesidade

- Idade avançada

Aproximadamente $70 \%$ dos casos de DAP podem ser explicados por FR estabelecidos, como idade avançada, hipertensão, dislipidemia, tabagismo e diabetes. ${ }^{8}$ Foi relatado que, para cada aumento de $1 \%$ na hemoglobina A1c, há um aumento correspondente de $26 \%$ no risco de DAP. ${ }^{9}$

A resistência à insulina foi identificada como um $\mathrm{FR}$ para DAP mesmo em indivíduos sem diabetes. ${ }^{10}$

A associação entre gênero e DAP é menos clara. A prevalência de DAP assintomática ou sintomática é ligeiramente maior nos homens do que nas mulheres e a incidência aumenta com o aumento da idade. ${ }^{11}$ As mulheres têm um estágio de ICM mais avançada na apresentação. ${ }^{12}$

A etnia negra aumenta o risco de DAP em duas vezes. ${ }^{11}$ Foi relatada também uma associação entre amputações maiores devido à DAP e comunidades desatendidas. ${ }^{13}$

O tabagismo tem sido fortemente associado à incidência de DAP e os fumantes pesados têm um risco quatro vezes maior de desenvolver $\mathrm{Cl}$ em comparação com não fumantes. ${ }^{11}$ A DAP compartilha FR com outras DCV como a DAC, porém alguns deles como o tabagismo estão mais poderosamente associados à DAP do que outras DCV.

\section{FISIOPATOLOGIA}

A DAP é uma manifestação da aterosclerose sistêmica que ocorre em virtude do estreitamento ou obstrução dos vasos sanguíneos arteriais, responsáveis por levar o sangue para nutrir as extremidades como braços e pernas, sendo mais comum o acometimento nos membros inferiores do que nos superiores. ${ }^{4}$ A DAP é causada principalmente por aterosclerose e trombose associadas nas artérias dos membros inferiores, levando à isquemia de órgãos finais. Outras causas incluem vasculites e trombose in situ relacionadas a estados hipercoaguláveis. ${ }^{4}$

A fisiopatologia da DAP induzida por aterotrombose é complexa e envolve um grande número de células, proteínas e caminhos. As células importantes que contribuem ou controlam o desenvolvimento da aterotrombose incluem células endoteliais vasculares, células musculares vasculares lisas, fibroblastos, plaquetas, células estaminais residentes, pericítas e células inflamatórias. ${ }^{14}$ 
Em condições saudáveis, a resposta à isquemia gradualmente progressiva dos membros envolve a promoção da angiogênese e da arteriogênese na tentativa de aumentar o suprimento de sangue ao membro afetado. O remodelamento vascular, a inflamação e as vias apoptóticas também estão implicados na resposta isquêmica e estes podem, em parte, contribuir para a resolução do dano tecidual. Em pacientes com isquemia crítica das pernas, essas respostas compensatórias à isquemia são ineficazes. Como resultado, existe uma perfusão inadequada de tecido, disfunção endotelial, inflamação crônica e altos níveis de estresse oxidativo. Todas essas mudanças levam à lesão mitocondrial, geração de radicais livres, dano de fibras musculares, degeneração de miofibra e fibrose e danos nos tecidos, que podem se apresentar como gangrena. ${ }^{15}$

A arteriogênese é o processo de aumento das artérias colaterais pré-existentes para contribuir para a perfusão tecidual. ${ }^{16} \mathrm{~A}$ principal força motriz é o aumento do fluxo de sangue laminar ou à tensão de cisalhamento associada à redistribuição do fluxo como resultado da redução da pressão luminal a jusante. $\mathrm{O}$ aumento da tensão de cisalhamento promove a ampliação do vaso, que, por sua vez é estimulado pela ativação da sinalização de óxido nítrico e provavelmente por outras respostas de sinalização ao fluxo. Fatores de crescimento como o fator de crescimento endotelial vascular e o fator induzido pela hipóxia desempenham um papel neste processo. As metaloproteinases de matriz (MMPs) são biomarcadores circulantes significativos que desempenham um papel fundamental na iniciação, progressão e manifestações clínicas da DAP. ${ }^{17}$

As placas de ateroma ocorrem na superfície posterior das artérias das extremidades. Com a evolução, a placa pode envolver circunferencialmente a artéria em certas áreas, mais freqüentemente nas origens ou nas bifurcações das artérias. Outros locais incluem a artéria femoral comum, a artéria femoral superficial distal (canal de Hunter) e o tronco tibioperoneiro. Além do acúmulo de placas, surgem trombos que agravam a oclusão arterial.

\section{DIAGNÓSTICO}

A estratégia diagnóstica da DAP inclui exame físico minucioso, com investigação de sinais clínicos sugestivos, tais como, palpação de pulsos periféricos, frêmitos arteriais e alterações de pele no membro afetado, bem como a confirmação da gravidade da obstrução vascular. Para confirmar o diagnóstico de DAP, os achados do exame físico anormal devem ser confirmados com testes diagnósticos, geralmente com o índice tornozelo-braquial (ITB) como teste inicial. ${ }^{18}$

Pacientes com diagnóstico confirmado de DAP correm maior risco de estenose da artéria subclávia. Uma diferença de pressão arterial entre os braços > 15 a 20 mm Hg é anormal e sugere estenose da artéria subclávia (ou inominada). A aferição da pressão arterial (PA) em ambos os braços identifica o braço com maior pressão sistólica, um requisito para uma medição precisa do ITB. A identificação de pressões sanguíneas desiguais nos braços também permite uma medição mais precisa da PA no tratamento da hipertensão (ou seja, a PA é aferida no braço com medidas mais elevadas). ${ }^{18}$

Estudos prévios focavam principalmente na claudicação como marcador para DAP, porém, o ITB é uma ferramenta mais objetiva e de baixo custo; padrão ouro para o diagnóstico não invasivo. ${ }^{19}$
O ITB é a proporção da pressão arterial sistólica no tornozelo para a do braço. Um valor anormalmente baixo do ITB é indicativo de aterosclerose na perna. Um ITB $\leq 0,90$ é comumente usado tanto na prática clínica quanto na pesquisa epidemiológica para diagnosticar a DAP, tanto sintomática quanto assintomática. $\mathrm{Na} \mathrm{Cl}$ mostrou ter baixa sensibilidade, mas uma alta especificidade para um ITB anormal. ${ }^{2}$

Os valores normais do ITB estão entre 1 e 1,4. Valores iguais a 0,9 são considerados anormais. Um ITB > 1,4 indica que as artérias não são compressíveis e valores de 0,91 a 0,99 são considerados limítrofes. Um ITB $<0,9$ está associado a um aumento de duas a quatro vezes o risco relativo de eventos cardiovasculares e mortalidade por todas as causas. Para melhorar a estratificação do risco cardiovascular e modificá-lo, a American Diabetes Association (ADA) recomenda o rastreamento pelo ITB para pacientes com mais de 50 anos com diabetes e a American Heart Association (AHA) recomenda a seleção de todos os pacientes com 65 anos ou mais e aqueles com idade $\geq 50$ anos e história de diabetes ou tabagismo. O rastreamento da DAP assintomática, pelo ITB, pode ser um importante aliado na estratificação do risco cardiovascular, especialmente nos pacientes de risco intermediário porém como não há evidências de que a triagem leva a menos eventos cardiovasculares ou diminui a mortalidade por todas as causas, a Força-Tarefa dos Serviços Preventivos dos EUA não a recomenda. ${ }^{20}$

A associação de medidas do ITB com os testes de caminhada melhora a avaliação do grau de acometimento do paciente com DAP, por associar medida inferencial de integridade de fluxo sanguíneo com capacidade funcional. Com a vasodilatação e a incapacidade de aumentar o fluxo depois do nível de obstrução aterosclerótica, o ITB medido após a atividade física cai em relação aos níveis de repouso. A redução da pressão sistólica após o exercício é um dos indicadores mais sensitivos para diagnostico de estenose hemodinamicamente significativa. Uma redução de $20 \%$ ou mais no ITB com exercício sugere DAP e pode identificar pacientes com risco aumentado de mortalidade. Uma análise transversal com duzentos e sessenta e cinco participantes do Estudo de População de San Diego com um ITB em repouso e após exercício, documentou a relação entre a mudança nas variáveis do ITB e as variáveis de risco múltiplo. A idade média dos participantes foi de 71,8 anos, e $80,4 \%$ eram do sexo feminino. Em repouso, o ITB médio foi 1,04 (DP 0,04) antes e 0,94 (DP 0,13) após o exercício; uma diminuição média de 9,5\%. Nas análises da mudança de ITB como variável contínua; maior idade, qualquer história de tabagismo e diagnóstico de doença pulmonar obstrutiva crônica (DPOC) estiveram associadas com uma diminuição significativa no ITB ao exercício. As análises categóricas dos FR associados a uma redução do ITB $\geq 20 \%$ com o exercício confirmaram esses resultados. A insuficiência cardíaca congestiva foi associada a um ITB aumentado com o exercício apenas em análises contínuas de alterações do ITB. A idade avançada, tabagismo e DPOC foram associados de forma independente e significativa com maior diminuição do ITB com o exercício podendo auxiliar na identificação de pessoas com DAP subclínico. ${ }^{21}$

Em pacientes idosos, devido a adaptações musculares e hemodinâmicas, circulação periférica, menor mobilidade 
por outras causas, sintomatologia infrequente, pode ser necessário o diagnóstico adequado por meio de exame de rastreio pelo ITB. ${ }^{21}$

O aumento da rigidez arterial resulta da elasticidade reduzida da parede arterial e é um preditor independente para o risco cardiovascular. O padrão-ouro para avaliação da rigidez arterial é a velocidade da onda de pulso (VOP) carotídeo-femoral. Outros parâmetros, como a pressão de pulso central aórtica e o índice de aumento aórtico (augmentation índex), são marcadores indiretos, de suporte da rigidez arterial, mas fornecem informações adicionais sobre as características da reflexão da onda. A DAP associa-se com hipertensão sistólica, aumento da rigidez arterial, distúrbio da reflexão da onda de pulso e prognóstico, dependendo do ITB. ${ }^{22}$

Os exames não invasivos da fisiologia vascular desempenham um papel importante no diagnóstico e na caracterização da DAP. Estes estudos avaliam os parâmetros fisiológicos do fluxo sanguíneo através de pressões arteriais segmentares, formas de onda Doppler e gravações de volume de pulso. Juntos, eles compõem um poderoso conjunto de ferramentas para definir a funcionalidade do sistema arterial, definir o local da doença e fornecer dados prognósticos. ${ }^{23}$

O exame inicial na investigação da DAP é frequentemente o ultrassom duplex (USD) tanto para o rastreamento como para o diagnóstico. O USD inclui as modalidades de ecografia modo B, onda pulsada, contínua, Doppler colorido de potência para detectar e localizar lesões vasculares e quantificar a sua extensão e gravidade através de critérios de velocidade. Técnicas mais recentes, como imagens de fluxo ou ecografia tridimensional (3D) ao vivo, bem como o uso de agentes de contraste de ultrassom, melhoram ainda mais o desempenho do USD, embora o seu uso ainda seja limitado. O USD pode detectar doença arterial subclínica (por exemplo, placa carotídea), o que é importante para a avaliação do risco $\mathrm{CV}^{23}$

A angiotomografia computadorizada (ATC) ou a angioressonância magnética (ARM) são reservadas para detaIhamento anatômico quando há previsão de intervenção cirúrgica ou endovascular. ${ }^{24}$

A ATC por multidetectores na imagem de vasos e órgãos tem um curto tempo de exame com artefatos de movimento e respiração reduzidos. As vantagens do ATC incluem rápida aquisição não invasiva, ampla disponibilidade, alta resolução e reformatação 3D. Semelhante ao USD e a ARM, a ATC exibe um "mapa" da vascularização, essencial para a determinação de estratégias intervencionistas. As desvantagens do ATC incluem a falta de dados funcionais e hemodinâmicos, a exposição à radiação e o uso de agentes de contraste iodados, que devem ser limitados no caso da doença renal crônica (DRC), com precauções em caso de alergias. A nefrotoxicidade pode ser limitada pela minimização do volume do agente de contraste e pela garantia de hidratação adequada antes e depois da aquisição das imagens. O benefício do uso da acetilcisteína para limitar a nefrotoxicidade é incerto. Estudos recentes sugeriram que as estatinas ou o bicarbonato de sódio podem prevenir a nefrotoxicidade do agente de contraste. Pesquisas adicionais são necessárias. ${ }^{24}$

A ARM é utilizada para imagem da artéria periférica utilizando técnicas de contraste (gadolínio) e não contraste (isto é, contraste de fase e sequências de tempo). Estas últimas técnicas possuem resolução inferior e são suscetíveis a artefatos, limitando a sua interpretação, mas são uma alternativa valiosa para uso em pacientes com DRC leve a moderada. Em comparação com o ATC, a ARM não precisa de contraste de iodo e tem maior resolução de tecido mole; entretanto, os artefatos de movimento são mais frequentes e as contraindicações incluem marcapassos e cardioversores-desfibriladores implantáveis (CDIs), [exceto ressonância magnética (RM) marca-passos condicionais e compatíveis, CDIs e derivações], claustrofobia e DRC grave. Neste último caso, o risco de fibrose sistêmica nefrogênica após a administração do gadolínio não deve ser subestimado. As calcificações vasculares, que podem afetar os procedimentos de revascularização, podem ser subestimadas. Os stents endovasculares não são avaliados pela ressonância magnética. ${ }^{24}$

O USD, a ATC e a ARM das extremidades inferiores são úteis para diagnosticar a localização anatômica e a gravidade da estenose para pacientes com DAP sintomáticos em quem a revascularização é considerada, porém não devem ser utilizadas para a avaliação anatômica de pacientes assintomáticos com DAP. ${ }^{18,24}$

A arteriografia por punção direta detecta o número de artérias com qualquer segmento opacificado. A arteriografia é pouco utilizada só para o diagnóstico, estando reservada para os casos de tratamento endovascular. ${ }^{24} \mathrm{~A}$ angiografia invasiva é útil para pacientes com ICM nos quais a revascularização é considerada e é razoável para pacientes com claudicação que limita o estilo de vida com resposta inadequada ao tratamento médico para quem a revascularização é considerada. Não deve ser realizada para a avaliação anatômica de pacientes com DAP assintomática. ${ }^{18}$ Pode ser utilizada no pré-operatório para planejamento da conduta e como ferramenta diagnóstico-terapêutica ao mesmo tempo em pacientes com diabetes.

Existe uma série de limitações para a utilização de marcadores circulantes atualmente identificados. Há pouco consenso sobre quais deles estão consistentemente associados à DAP e há desafios definidos na identificação de marcadores específicos da DAP. ${ }^{4}$ Embora novos marcadores, como a atividade da Rho-quinase, a glicoproteína-39 da cartilagem humana, o TWEAK e o PON-3, tenham sido associados à presença de DAP, sua validade como marcador confiável não está clara, pois esses relatos individuais não são frequentemente replicados por outros estudos. Alguns marcadores de inflamação, como B2M, hsCRP e interleucinas, estresse oxidativo, como $\mathrm{NO}$ e NOX-2, remodelamento ECM, como TGF- $\mathrm{1,} \mathrm{TSP-1} \mathrm{e} \mathrm{VEGF,} \mathrm{e} \mathrm{populações} \mathrm{de} \mathrm{células} \mathrm{progenitoras}$ circulantes têm sido consistentemente associados com presença de DAP. Da mesma forma, marcadores de inflamação, como hsCRP e sVCAM-1, estresse oxidativo, como MPO e NT-pro-BNP, remodelamento ECM, como o VEGF, as isoformas de VEGF-A e MMPs, foram consistentemente associados à gravidade da DAP. ${ }^{4}$ Nenhum biomarcador mostrou-se atualmente suficientemente robusto para ser incorporado na prática clínica. Pode ser necessária uma combinação de fatores de risco e dados do marcador circulante. Mesmo que uma série de marcadores circulantes atualmente identificados sejam promissores, são necessários estudos mais amplos focados no valor clínico destes marcadores em relação aos preditores de risco já existentes. ${ }^{4}$ 


\section{TRATAMENTO}

Deve-se antecipar o diagnóstico e o tratamento da DAP, já que sua presença indica processo aterosclerótico disseminado, comprometendo a sobrevida do indivíduo. Os dois principais objetivos do tratamento da DAP são de reduzir os sintomas onerosos e o risco cardiovascular (CV) aumentado. A aterosclerose é doença sistêmica e, como tal, todo paciente com DAP deve receber tratamento antiaterosclerose ${ }^{24}$.

O controle dos FR constitui seguramente a parte mais importante do tratamento médico. A interrupção do hábito de fumar é fundamental, ${ }^{18,24}$ além do controle do peso, do etilismo e do sedentarismo. O uso de anti-hipertensivos, estatinas, antiagregantes plaquetários são fundamentais no tratamento da DAP. Os pacientes diabéticos devem ter um controle glicêmico estrito, ${ }^{24}$ serem bem acompanhados, quando possível, por equipe multidisciplinar na prevenção e no tratamento do pé diabético.

No tratamento não farmacológico da $\mathrm{Cl}$ tem sido relevante o papel do exercício. O exercício não apenas proporciona melhora na capacidade de deambulação, mas também na qualidade de vida. O exercício é uma opção de tratamento igualmente eficaz para melhorar a distância de caminhada; uma metanálise de estudos randomizados mostrou que o exercício aumentou a distância de marcha em 179 metros em média. Entretanto, para muitos destes pacientes existe a necessidade de uma reabilitação com supervisão devido a forte associação da DAP com a doença cardíaca ou cerebral. ${ }^{29}$ Programas de exercícios supervisionados, embora não sejam amplamente implementados, foram estabelecidos como formas efetivas de aumentar a distância livre para a dor entre pacientes com $\mathrm{Cl}^{25}$

Além do controle dos principais $\mathrm{FR}$, alguns fármacos podem ser úteis, especialmente para redução do risco cardiovascular global. Algumas drogas tradicionalmente utilizadas no alívio da Cl têm um efeito modesto e o campo de aplicação é limitado de acordo com as diretrizes atuais. ${ }^{18,24}$

Os agentes antiplaquetários são efetivos em reduzir o risco de eventos isquêmicos fatais e não fatais em pacientes com DAP. A terapia antiplaquetária com aspirina isoladamente (intervalo de 75-325 mg por dia) ou clopidogrel (75 mg por dia) é recomendável para reduzir o IM, AVC e morte vascular em pacientes com DAP sintomática. ${ }^{18} \mathrm{Em}$ pacientes assintomáticos com DAP (ITB $\leq 0,9)$, a terapia antiplaquetária é razoável para reduzir o risco de IM, AVC ou morte vascular. Em pacientes assintomáticos com ITB limítrofe $(0,91-0,99)$, a utilidade da terapia antiplaquetária para reduzir o risco de IM, AVC ou morte vascular não está definida. ${ }^{18}$

A eficácia da terapia antiplaquetária dupla (aspirina e clopidogrel) para reduzir o risco de eventos isquêmicos cardiovasculares em pacientes com DAP sintomática não está bem estabelecida. Pode ser razoável para reduzir o risco de eventos relacionados aos membros em pacientes com DAP sintomática após revascularização das extremidades inferiores. ${ }^{18}$

A recente diretriz da Sociedade Europeia de 2017 (ESC 2017) ${ }^{24}$ preconiza monoterapia em todos os pacientes com exceção da DAP assintomática, na qual não há indicação formal de antiplaquetários, dupla antiagregação por 30 dias após stent endovascular. Em pacientes com indicação para anticoagulação sistêmica (ex: FA) e portadores de DAP, podendo-se não associar antiagregante plaquetário, salvo se portador de stent. Um estudo sugere que na DAP o clopidogrel seria mais eficaz que aspirina em monoterapia. ${ }^{24}$

No tratamento da $\mathrm{Cl}$ as drogas hemorreológicas são utilizadas extensamente, dentre elas destacam-se a pentoxilfilina e o cilostazol. Atualmente, estudos evidenciam a superioridade do cilostazol na intervenção. O cilostazol, nibidor da fosfodiesterase-3, inibe a agregação plaquetária e causa vasodilatação, além de melhora no perfil lipídico e tem se mostrado mais promissor e, na dose de $100 \mathrm{mg}$ duas vezes ao dia; demonstrou ser superior não apenas ao placebo, mas também à pentoxifilina. O cilostazol é uma terapia eficaz para melhorar os sintomas e aumentar a distância a pé em pacientes com claudicação. No entanto, os efeitos colaterais incluem dor de cabeça, diarréia, tonturas e palpitações e em um ensaio, 20\% dos pacientes descontinuaram o cilostazol em 3 meses. ${ }^{18}$ A pentoxifilina tem sido questionada nos últimos anos, quando não se mostrou mais efetiva do que o placebo em um estudo controlado e randomizado, enquanto um benefício foi demonstrado em metanálise de estudos menores. A pentoxifilina oral (400 mg 2x/dia) pode ser empregada, porém não é eficaz para o tratamento da claudicação. ${ }^{18}$

O tratamento com uma estatina é indicado para todos os pacientes com DAP devendo ser utilizada a dose máxima, pois pacientes com DAP são equivalentes de alto risco de doença cardiovascular. O alvo recomendado pela diretriz ESC de 2017: LDL < $70 \mathrm{mg} / \mathrm{dl}$ e pelo menos 50\% de redução se LDL basal entre 70-135 mg/dl. Em estudos observacionais e ensaios clínicos randomizados (ECR) em pacientes com DAP (de casos assintomáticos a graves), o tratamento com estatinas tem provocado reduções na mortalidade por todas as causas e eventos CV. No registro Reduction of Atherothrombosis for Continued Healt (REACH), entre pacientes com DAP, o uso de estatina foi associado a uma redução de $17 \%$ nas taxas de eventos cardiovasculares adversos. Mesmo nos estágios mais avançados da doença, a terapia com estatina está associada a menores taxas de mortalidade em um ano de eventos adversos CV. O tratamento combinado com ezetimiba em pacientes selecionados também é benéfico. Em um ensaio randomizado, o bezafibrato não mostrou benefício em relação ao placebo para reduzir eventos coronarianos e cerebrovasculares em pacientes com DAP. Nos pacientes com DAC, as estatinas reduzem o risco de derrame. Recentemente, o estudo de Fourier demonstrou os benefícios adicionais do evolocumabe, um anticorpo monoclonal que inibe a proproteína convertase subtilisina / kexina tipo 9 para reduzir os eventos CV em pacientes com doença aterosclerótica sobre estatinas isoladamente. Os resultados foram consistentes no subgrupo de 1505 pacientes com DAP sozinhos, porém mais resultados são aquardados. ${ }^{24}$

A terapia de quelação (por exemplo, ácido etilenodiaminotetracético não é benéfica para o tratamento da claudicação. ${ }^{18}$

Não se recomenda a suplementação vitamínica de complexos B para níveis mais baixos de homocisteína para prevenção de eventos cardiovasculares em pacientes com DAP. ${ }^{18}$

Outras drogas vasodilatadoras, terapia de reposição hormonal não se mostraram efetivas para o tratamento da claudicação ou, ainda, não foram testadas em grandes estudos controlados e randomizados. ${ }^{18}$ 
Pacientes com DAP devem ter uma vacinação anual contra influenza. ${ }^{18}$

A utilidade da anticoagulação para melhorar a permeabilidade após a veia autógena das extremidades inferiores ou o bypass protético é incerto. A anticoagulação não deve ser usada para reduzir o risco de eventos isquêmicos cardiovasculares em pacientes com DAP. ${ }^{18}$

A terapia anti-hipertensiva deve ser administrada a pacientes com hipertensão e DAP para reduzir o risco de IM, AVC, insuficiência cardíaca e morte cardiovascular. O uso de inibidores da enzima conversora da angiotensina (IECA) ou bloqueadores dos receptores da angiotensina (BRA) pode ser eficaz para reduzir o risco de eventos isquêmicos cardiovasculares em pacientes com DAP. ${ }^{18}$ IECA e BRA são drogas de escolha na HAS. Não há contra indicação aos betabloqueadores mesmo em pacientes com DAP. Meta PA $\leq 140 \times 90 \mathrm{mmHg}$ (em diabéticos, considerar PA diastólica $\leq 85 \mathrm{mmHg}$ ). ${ }^{24}$

Outras terapias médicas são aplicadas para controlar a dor, tratar a infecção e promover a cicatrização de úlceras. O paciente queixando-se de $\mathrm{Cl}$, cuja distância útil seja limitante às atividades profissionais ou mesmo de $\operatorname{lazer}^{20}$ e pacientes com ICM (dor em repouso, gangrena ou úlcera isquêmica), ou seja, em situações em que há ameaça à viabilidade dos membros, a cirurgia de revascularização ou a terapia endovascular (TEV) são claramente indicados e serão escolhidos de acordo com as condições gerais do paciente e locais dos segmentos arteriais atingidos. ${ }^{20}$

As terapias cirúrgicas endovasculares e abertas proporcionam resultados semelhantes para ICM. Uma meta-análise recente de 23 estudos não relatou diferença na sobrevida livre de amputação aos três anos (OR, 1,22, IC 95\%: 0,84-1,77) e mortalidade por todas as causas (OR, 1,07, IC 95\%: 0,73-1,56) em pacientes com ICM tratados por TEV ou revascularização cirúrgica. ${ }^{26}$

A TEV oferece alívio dos sintomas e maior qualidade de vida, é um método eficaz para pacientes com estenose de alto grau dos segmentos arteriais dos membros proximais. Os avanços na técnica e tecnologia aumentaram a viabilidade e praticidade da TEV, que agora representa o modo preferido de revascularização em muitos centros em todo o mundo. ${ }^{27}$

O tratamento cirúrgico pode requerer a amputação em alguns pacientes; felizmente o número é pequeno, comparado ao número de pacientes com sintomas. Os objetivos da cirurgia de amputação são: remover a gangrena, tecidos necróticos ou infectados; aliviar a dor; obter a cicatrização primária da porção mais distal possível e conseguir a reabilitação máxima após a amputação. ${ }^{20}$

Existe um interesse atual no desenvolvimento de novas terapias para melhorar a arteriogênese (formação colateral) e/ou angiogênese (formação capilar) em pacientes com DAP. ${ }^{28}$ Atualmente, estão sendo investigadas várias abordagens, incluindo terapias genéticas e terapias baseadas em células. ${ }^{29}$ No estudo RESTORE-CLI, o uso de células de reparo de tecidos em pacientes com DAP, foi relatado que a administração de células mononucleares de medula óssea derivadas do paciente levou a uma melhoria de sobrevivência sem amputação de $32 \%{ }^{30}$ Essas novas abordagens terapêuticas são estratégias promissoras para pacientes com isquemia crítica que não são candidatos ideais para procedimentos tradicionais de revascularização. As terapias potenciais destinadas a acelerar a arteriogênese e a angiogênese durante a isquemia estão sendo intensamente investigadas, embora a maioria dessas intervenções não tenha evoluído para uma fase em que estejam prontas para uso clínico generalizado.

\section{TRATAMENTO ANTI-HIPERTENSIVO}

Embora não haja desentendimento de que o tratamento anti-hipertensivo melhore os desfechos clínicos, o nível de PA para o melhor benefício ainda não está claro. Entre indivíduos sem doença cardiovascular aparente, níveis de PA progressivamente menores estão associados a resultados cardiovasculares melhores até um nível de pelo menos 115/75 mm Hg; no entanto, no grande grupo de pacientes com comorbidades como DAC, diabetes e DAP, ainda há um debate sobre como a PA deve ser reduzida. Isto se deve à adequação da perfusão miocárdica a esses níveis mais baixos de PA, pois dados de vários estudos observacionais sugeriram um aumento nos eventos isquêmicos cardíacos nesses níveis mais baixos. Muitos desses pacientes também apresentam disfunção microvascular coronária, o que poderia potencializar quaisquer efeitos nocivos associados à PA muito baixa. ${ }^{31}$

Dados do estudo International VErapamil-SR / Trandolapril (INVEST) sugeriu que deve-se ter cautela para evitar uma queda da pressão arterial sistólica (PAS) abaixo de 110-120 $\mathrm{mmHg}$, uma vez que a relação em forma de J entre PAS e eventos CV foi relatada em pacientes com DAP. ${ }^{32}$ (Figura 1)

Ensaio clínico randomizado, o estudo INVEST incluiu 2699 pacientes hipertensos com DAP concomitante e DAC seguidos por uma média de 2,7 anos (60 970 pacientes-ano). O desfecho primário; morte por todas as causas, IM não fatal ou AVC não fatal ocorreram em 16,3\% dos pacientes com DAP versus $9,2 \%$ sem DAP (razão de risco ajustada: 1,26 [IC 95\%: 1,13 a 1,40]; P $<0,0001)$. O resultado primário ocorreu com menor frequência entre os pacientes com DAP tratados com uma PAS média de 135 a 145 mm Hg e uma pressão arterial diastólica (PAD) média de 60 a $90 \mathrm{~mm} \mathrm{Hg}$. Os pacientes com DAP mostraram uma relação em forma de J com a PAS e o desfecho primário, embora indivíduos sem DAP não o tenham feito. O estudo sugeriu que os pacientes com DAP podem exigir uma PA alvo diferente daquelas sem DAP. ${ }^{32}$

No estudo INVEST, não houve diferença nos resultados de eventos CV entre a estratégia do verapamil mais

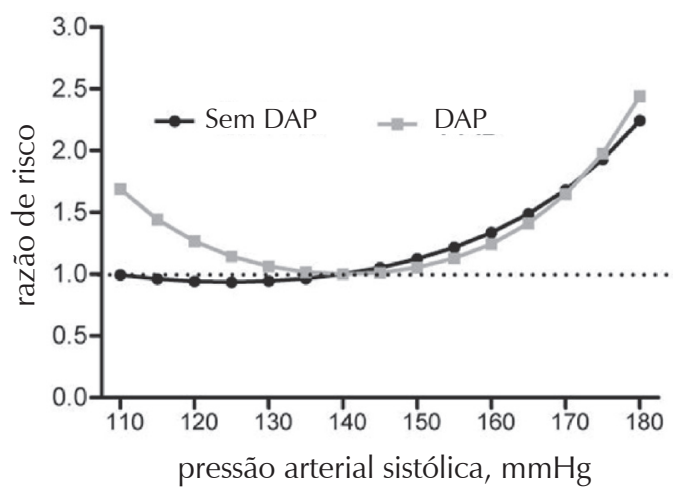

Adaptado de Bavry AA, et al.32

Figura 1. A figura ilustra um aumento desproporcional no risco de desfechos adversos entre pacientes com baixa pressão arterial sistólica que apresentam doença arterial periférica (DAP) concomitante e doença arterial coronariana. 
trandolapril versus a estratégia de atenolol mais hidroclorotiazida. ${ }^{32}$ Algumas classes, porém, podem ser preferidas de acordo com as comorbidades.

Evidências sobre o uso de vários medicamentos anti-hipertensivos em pessoas com DAP são pobres, de modo que ainda não sabemos se benefícios ou riscos significativos se acumulam. No entanto, a falta de dados que examinam especificamente os resultados em pacientes com DAP não deve prejudicar a evidência esmagadora sobre o benefício do tratamento da hipertensão e redução da PA. ${ }^{33}$ Uma revisão da Cochrane concluiu que houve falta de evidência sobre a eficácia da medicação anti-hipertensiva em pacientes com DAP, mas não realizou meta-análise ou meta-regressão para avaliar definitivamente isso. ${ }^{33}$

O efeito da estratégia de tratamento da hipertensão entre pacientes com DAP foi avaliado em uma revisão sistemática e meta-análise recente, realizada para avaliar se os medicamentos anti-hipertensivos pioravam a isquemia das pernas em pacientes com DAP, incluindo distância máxima a pé, distância de caminhada livre de dor e ITB. Este estudo sugeriu que o tratamento anti-hipertensivo não piora, mas pode melhorar a isquemia das pernas em pacientes com DAP. ${ }^{34}$

Entre os pacientes com DAP, a incidência do desfecho primário não foi significativamente diferente entre as estratégias de tratamento, embora as tendências favoreçam a estratégia de antagonista de cálcio, em 15,5\% com uma estratégia baseada em antagonista de cálcio versus 17,1\% com uma estratégia baseada em $\beta$ bloqueadores (HR : 0,89 [IC 95\%: 0,74 a 1,07]; $\mathrm{P}=0,21$ ). Do mesmo modo, um evento no resultado primário ou primeira ocorrência de um procedimento vascular não foi diferente, em 17,5\% contra 18,2\% (HR: 0,94 [IC 95\%: 0,77 a $1,13] ; P=0,50)$. No entanto, um evento no desfecho primário ou qualidade de vida pobre / justa foi de $36,5 \%$ entre aqueles que receberam a estratégia de antagonista de cálcio versus $41,0 \%$ entre aqueles que receberam a estratégia de $\beta$ bloqueador (HR: 0,87 [IC 95\%: 0,77 a 0,99]; P = 0,031). ${ }^{34}$ (Figura 2)

A recente diretriz da ESC 2017 recomenda nestes pacientes uma meta de PA inferior a 140/90 $\mathrm{mmHg}$, com exceção dos pacientes com diabetes, para os quais uma PAD $\leq 85$ $\mathrm{mmHg}$ é considerada segura. Em pacientes idosos e frágeis,

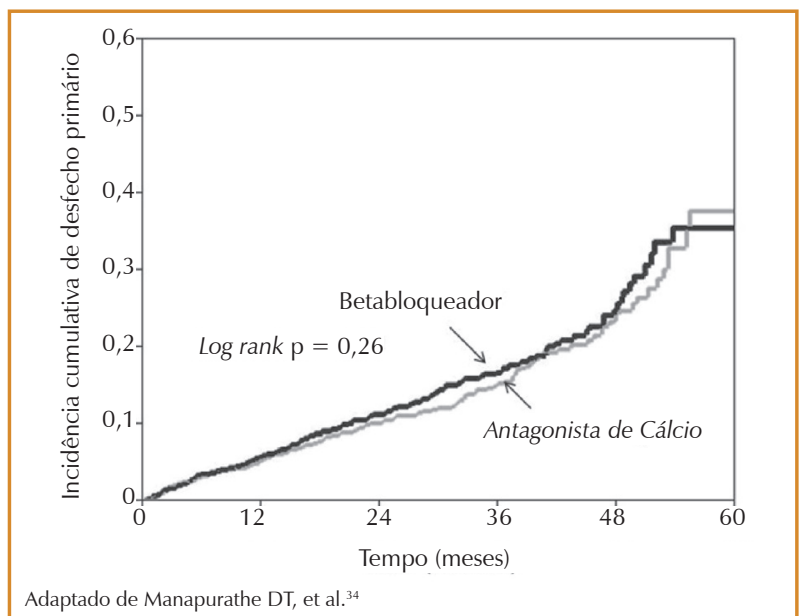

Figura 2. Curva de Kaplan-Meier para o desfecho primário em pacientes com DAP estratificados por regime anti-hipertensivo. esses níveis devem ser alcançados somente se bem tolerados, sem hipotensão ortostática. ${ }^{24}$

Diuréticos, $\beta$ bloqueadores, antagonistas do cálcio, inibidores da enzima conversora da angiotensina (IECA) e bloqueadores dos receptores da angiotensina (BRA) são todos adequados para o tratamento anti-hipertensivo, como monoterapia ou em diferentes combinações. A terapia anti-hipertensiva deve ser administrada a pacientes com hipertensão e DAP para reduzir o risco de IM, AVC, insuficiência cardíaca e morte cardiovascular. ${ }^{18,24}$

O uso de iECA ou BRA é eficaz para reduzir o risco de eventos isquêmicos $C V$ em pacientes com DAP e são drogas de escolha na HA. O Heart Outcomes Prevention Trial (HOPE) e o estudo Ongoing Telmisartan Alone and in Combination with Ramipril Global Endpoint Trial (ONTARGET) demonstraram que os IECA e BRA reduzem significativamente os eventos $\mathrm{CV}$ em doentes com DAP. De acordo com estes ensaios, os IECA ou BRA são recomendados para prevenção secundária, mesmo em pacientes com isquemia crônica que ameace os membros, pois nesse subgrupo de pacientes, houve diminuição dos eventos $\mathrm{CV}$ adversos maiores e mortalidade sem qualquer efeito nos desfechos dos membros. ${ }^{24}$

É importante ressaltar que nas diretrizes da AHA/ACC 2016 e ESC 2017 os $\beta$ bloqueadores não foram contraindicados em pacientes com DAP, uma vez que não alteram a capacidade de deambulação em pacientes com DAP leve a moderada. Em um estudo observacional, pacientes com DAP e IM prévio e tomando $\beta$ bloqueadores tiveram uma redução significativa de $53 \%$ do risco de eventos coronarianos aos 32 meses. Entretanto, eles devem ser cuidadosamente prescritos para pacientes com isquemia crônica dos membros. ${ }^{18,24}$

Ensaios multicêntricos maiores com períodos de tratamento anti-hipertensivos mais longos são necessários para esclarecer o efeito de anti-hipertensivos na isquemia das pernas em pacientes com DAP.

\section{PONTOS-CHAVE}

- A doença arterial periférica (DAP) é uma condição comórbida comum frequentemente encontrada em pacientes com hipertensão. - O índice tornozelo braquial (ITB) é uma boa ferramenta de triagem para detecção de DAP.

- A modificação do estilo de vida e a terapia antiplaquetária continuam a ser a terapia padrão para o gerenciamento do DAP. - A doença arterial periférica (DAP) é um importante marcador de doença cardiovascular geral.

- Todos os pacientes com DAP devem receber modificações agressivas de fatores de risco cardiovasculares.

- Pacientes com diabetes e fumantes apresentam risco particularmente alto de desenvolver isquemia de membro crítico. - A terapia anti-hipertensiva deve ser administrada a pacientes com hipertensão e DAP para reduzir o risco de IM, AVC, insuficiência cardíaca e morte cardiovascular.

- A redução da pressão arterial sistólica (PAS) reduz eventos cardiovasculares e a meta atual para tratamento na DAP é de PA $\leq 140 \times 90$ mmHg (em diabéticos, considerar PA diastólica $\leq 85 \mathrm{mmHg}$ ).

- O uso de inibidores da enzima conversora da angiotensina (iECA) ou bloqueadores dos receptores da angiotensina (BRA) são eficazes e são drogas de escolha na HA. Não há contra indicação aos betabloqueadores nestes pacientes. 


\section{REFERÊNCIAS}

1. Lim SS, Vos T, Flaxman AD, Danaei G, et al. A comparative risk assessment of burden of disease and injury attributable to 67 risk factors and risk factor clusters in 21 regions, 1990-2010: a systematic analysis for the Global Burden of Disease Study 2010. Lancet. 2013, 380(9859):2224-60. doi: 10.1016/S0140-6736(12)61766-8

2. Criqui $\mathrm{MH}$, Aboyans V. Epidemiology of peripheral artery disease. Circ Res. 2015;116(9):1509-26. doi: 10.1161/CIRCRESAHA.116.303849.

3. Fowkes FG, Rudan D, Rudan I et al. Comparison of global estimates of prevalence and risk factors for peripheral artery disease in 2000 and 2010 : a systematic review and analysis. Lancet. 2019;382(9901):1329-40. doi: 10.1016/S0140-6736(13)61249-0.

4. Krishna SM, Moxon JV, Golledge J. A review of the pathophysiology and potential biomarkers for peripheral artery disease. Int J Mol Sci. 2015;16(5):11294-322. doi: 10.3390/ijms160511294.

5. Subherwal S, Patel MR, Kober L,et al. Peripheral artery disease is a coronary heart disease risk equivalent among both men and women: Results from a nationwide study. Eur J Prev. Cardiol. 2015;22:317-25.

6. Walker TG. Acute limb ischemia. Tech Vasc Interv Radiol. 2009; 12:117-29.

7. Wattanakit K, Folsom AR, Selvin E, et al. Risk factors for peripheral arterial disease incidence in persons with diabetes: the Atherosclerosis Risk in Communities (ARIC) Study. Atherosclerosis. 2005 Jun; 180(2):389-97. doi: 10.1016/j.atherosclerosis.2004.11.024. Epub 2005 Jan 25.

8. Meijer WT, Grobbee DE, Hunink MG, Hofman A, Hoes AW. Determinants of peripheral arterial disease in the elderly: The Rotterdam study. Arch Intern Med. 2000;160:2934-8.

9. Selvin E, Marinopoulos S, Berkenblit G, et al. Meta-analysis: Glycosylated hemoglobin and cardiovascular disease in diabetes mellitus. Ann Intern Med 2004; 141:421-31.

10. Muntner P, Wildman RP, Reynolds K, Desalvo KB, Chen J, Fonseca V. Relationship between $\mathrm{HbA} 1 \mathrm{c}$ level and peripheral arterial disease. Diabetes Care. 2005;28:1981-87. doi: 10.2337/diacare.28.8.1981.

11. Norgren L, Hiatt WR, Dormandy JÁ, Nehler MR, Harris KA, Fowkes FG. TASC II Working Group. Inter-society consensus for the management of peripheral arterial disease (TASC II). J Vasc Surg. 2007;45:S5-S67. doi: 10.1016/j.jvs.2006.12.037.

12. Lo RC, Bensley RP, Dahlberg SE, et at. Presentation, treatment, and outcome differences between men and women undergoing revascularization or amputation for lower extremity peripheral arterial disease. J Vasc Surg. 2014; 59:409-18 .e3. doi: 10.1016/j.jvs.2013.07.114.

13. McGinigle KL, Kalbaugh CA, Marston WA. Living in a medically underserved county is an independent risk factor for major limb amputation. J Vasc Surg. 2014;59:737-41. doi: 10.1016/j.jvs.2013.09.037.

14. Badimon L, Vilahur G. Thrombosis formation on atherosclerotic lesions and plaque rupture. J Intern Med. 2014;276:618-32. doi: 10.1111/joim.12296.

15. Koutakis P, Myers SA, Cluff K, et al. Abnormal myofiber morphology and limb dysfunction in claudication. J Surg Res. $2015 ; 196(1): 172-9$. doi: 10.1016/j.jss.2015.02.011.

16. Scholz D, Cai WJ., Schaper W. Arteriogenesis, a new concept of vascular adaptation in occlusive disease. Angiogenesis. 2001;4(4):247-57. doi: 10.1023/a:1016094004084

17. Giagtzidis I, Karkos C, Pitoulias G, Papazoglou K. Matrix metalloproteinases and peripheral arterial disease. Int Angiol. $2015 ; 34(3): 195-201$

18. Gerhard-Herman MD, Gornik HL, Barrett C, et al. 2016 AHA/ACC Guideline on the Management of Patients With Lower Extremity Peripheral Artery Disease: Executive Summary: A Report of the American College of Cardiology/American Heart Association Task Force on Clinical Practice Guidelines. Circulation. 2017; 135(12): e686-e725. doi:10.1161/ CIR.0000000000000470.

19. Crawford F, Welch K, Andras A, Chappell FM. Ankle brachial index for the diagnosis of lower limb peripheral arterial disease. Cochrane Database Syst Rev. 2016;9:CD010680.

20. Hennion DR, Siano KA. Diagnosis and treatment of peripheral arterial disease. Am Fam Physician. 2013;88(5):306-10.

21. Alqahtani KM, Bhangoo M, Vaida F, Denenberg JO, Allison MA, Criqu $\mathrm{MH}$. Predictors of Change in the Ankle Brachial Index with Exercise. Eur Vasc Endovasc Surg. 2018. pii: S1078-5884(17) 30729-3. doi: 10.1016/j. ejvs.2017.12.004

22. Husmann M, Jacomella $V$, Thalhammer C, Amann-Vesti BR. Markers of arterial stiffness in peripheral arterial disease. Vasa. 2015 Sep;44(5):341-8. doi: 10.1024/0301-1526/a000452.

23. Sibley RC, Reis SP, MacFarlane J, Reddick MA, Kalva SP, Sutphin PD. Noninvasive Physiologic Vascular Studies: A Guide to Diagnosing Peripheral Arterial Disease. Radiographics. 2017; 37(1):346-57. doi: 10.1148/ rg.2017160044.

24. Aboyans V, Ricco JB, Bartelink MEL, et al. 2017 ESC Guidelines on the Diagnosis and Treatment of Peripheral Arterial Diseases, in collaboration with the European Society for Vascular Surgery (ESVS): Document covering atherosclerotic disease of extracranial carotid and vertebral, mesenteric, renal, upper and lower extremity arteriesEndorsed by: the European Stroke Organization (ESO)The Task Force for the Diagnosis and Treatment of Peripheral Arterial Diseases of the European Society of Cardiology (ESC) and of the European Society for Vascular Surgery (ESVS). Eur Heart J. 2018;39(9):763-816. doi: 10.1093/eurheartj/ehx095.

25. Morris DR, Rodriguez AJ, Moxon JV, et al. Association of lower extremity performance with cardiovascular and all-cause mortality in patients with peripheral artery disease: A systematic review and meta-analysis. J Am Heart Assoc. 2014;3(4). pii: e001105. doi: 10.1161/JAHA.114.001105.

26. Jones WS, Dolor RJ, Hasselblad V, et al. Comparative effectiveness of endovascular and surgical revascularization for patients with peripheral artery disease and critical limb ischemia: Systematic review of revascularization in critical limb ischemia. Am HeartJ. 2014;167(4):489-498.e7. doi: 10.1016/j. ahj.2013.12.012.

27. Bunte MC, Shishehbor MH. Next Generation Endovascular Therapie in Peripheral Artery Disease. Prog Cardiovasc Dis. 2018. pii: S00330620(18)30055-0. doi: 10.1016/j.pcad.2018.03.003.

28. Lau JF, Weinberg MD, Olin JW. Peripheral artery disease. Part 1: clinical evaluation and noninvasive diagnosis. Nat Rev Cardiol. 2011;8(7):405-18. doi: 10.1038/nrcardio.2011.66.

29. Davies MG. Critical limb ischemia: Cell and molecular therapies for limb salvage. Methodist DeBakey Cardiovasc J. 2012;8(4):20-7. doi: 10.14797/ mdcj-8-4-20.

30. Powell RJ, Marston WA, Berceli AS, et al. Cellular therapy with ixmyelocel-T to treat critical limb ischemia: The randomized, double-blind, placebocontrolled RESTORE-CLI trial. Mol Ther 2012:20:1280-6. doi: 10.1038/ mt.2012.52.

31. Bavry AA, Pepine CJ. Treatment of Hypertension: Lower Is Better, Or Is It? Hypertension. 2012; 60(2): 281-2. doi: 10.1161/ HYPERTENSIONAHA.112.197632.

32. Bavry AA, Anderson RD, Gong Y, et al. Outcomes among hypertensive patients with concomitant peripheral and coronary artery disease: finding from the INternational VErapamil-SR/Trandolapril STudy. Hypertension. 2010;55:48-53. doi: 10.1161/HYPERTENSIONAHA.109.142240.

33. Lane DA, Lip GY. Treatment of hypertension in peripheral arteria disease. Cochrane Database Syst Rev. 2013;(12):CD003075. doi: $10.1002 / 14651858 . C D 003075$.

34. Manapurathe DT, Krishna S M, Dewdney B, Moxon JV, Biros E, Golledge J Effect of blood pressure lowering medications on leg ischemia in peripheral artery disease patients: A meta-analysis of randomised controlled trials. PLoS One. 2017;12(6): e0178713. doi: 10.1371/journal.pone.0178713. 